\title{
DETERMINACIÓN DEL MOMENTO ÓPTIMO ECONÓMICO DE BENEFICIO DE CUYES ALIMENTADOS CON ALFALFA $v$ s. UNA SUPLEMENTACIÓN CON AFRECHILLO
}

\author{
Ronald Jiménez A. ${ }^{1}$, Custodio Bojórquez R. ${ }^{1}$, Felipe San Martín H. ${ }^{2}$, \\ Fernando Carcelén $\mathrm{C}^{2}{ }^{2}$ Alfredo Pérez $\mathrm{S}^{3}$
}

\section{Abstrat}

The relationship between nutrition and optimal culling age of guinea pigs (Cavia porcellus) was determined at the El Mantaro Station of the Veterinary Institute for Tropical and High Altitude Research, IVITA. A total of 40 weaned guinea pigs (average age 14.5 days) were fed two diets: $\mathrm{T} 1$ alfalfa and $\mathrm{T} 2$ alfalfa plus wheat hulls (afrechillo) both fed ad libitum. A random block design, with sex defining the block, and 4 experimental units of 5 animals each, was utilized for both experiments. Optimal culling age, determined by regression analysis utilizing food consumption/live weight and age/live weight., was found to be 12.9 and 11.8 weeks for males, 12.9 and 12 weeks for females fed diets T1 and T2. Average consumption of dry matter/animal/day was 57 and $59.6 \mathrm{~g}$ for T1 and T2 males, and 53.6 and $59.3 \mathrm{~g}$ for $\mathrm{T} 1$ and $\mathrm{T} 2$ females, with average weight gains of 8.6 and $9.4 \mathrm{~g} / \mathrm{animal} / \mathrm{day}$ for males, and 7.5 and $9.2 \mathrm{~g} / \mathrm{animal} /$ day for females. Nutritional conversion indices were: 6.6 and 6.2 for males, and 7.0 and 6.4 for females, on diets $\mathrm{Tl}$ and T2. No statistically significant differences were found $(P>0.05)$ between the treatments in regard to sex, food consumption, weight gain or nutritional conversion. Production costs were lower for $\mathrm{T} 2$ than T1, averaging 4.08 versus 4.31 New Soles for males; 4.13 versus 4.32 for females, and the optimal age for culling was also reached approximately one week earlier among animals on the T2 combination alfalfa and wheat hull diet.

Key words: Guinea pig, culling, diet, alfalfa, afrechillo, wheat hulls.

\section{Resumen}

Se determinó la edad óptima de beneficio de cuyes sometidos a diferente regimen alimenticio en la Estación Experimental del IVITA - El Mantaro. Se utilizaron 40 cuyes destetados (edad promedio $14.5 \mathrm{~d}$ ) alimentados con dos dietas: T1 alfalfa y T2 alfalfa más afrechillo ambos suministrados ad libitum. Se utilizó un diseño de bloques al azar, con sexo como bloque, y cuatro unidades experimentales de cinco animales cada uno para ambos tratamientos. Se estimó la edad óptima de beneficio utilizando el análisis de regresión para consumo de alimento/peso vivo y edad/peso vivo, encontrándose para machos que la edad óptima de beneficio es de 12.9 y 11.8 semanas y hembras de 12.9 y 12 semanas para $\mathrm{T} 1$ y $\mathrm{T} 2$, respectivamente. El consumo promedio de materia seca/animal/ dia fue de $57.059 .6 \mathrm{~g}$ para $\mathrm{T} 1$ y $\mathrm{T} 2 \mathrm{en}$ machos, y 53.6 y $59.3 \mathrm{~g}$ para $\mathrm{T} 1 \mathrm{y} \mathrm{T} 2$ en hembras,

1 Estación Experimental El Mantaro - IVITA - FMV - UNMSM.

2 Laboratorio de Nutrición Animal IVITA - FMV - UNMSM. E.Mail: d170032@unmsm.edu.pe

3 Laboratorio de Producción Agropecuaria FMV - UNMSM. 
con una ganancia promedio diaria de peso de 8.6 y $9.4 \mathrm{~g}$ para machos y 7.5 y $9.2 \mathrm{~g}$ para hembras en los T1 y T2, respectivamente. No se encontraron diferencias ( $p>0.05$ ) entre tratamientos con respecto a sexo, consumo, ganancia de peso y conversión alimenticia. Los costos de producción fueron menores para T2 que T1 promediando 4.08 vs. 4.31 Nuevos Soles para machos; 4.13 vs. 4.32 para hembras. La edad óptima para beneficio se alcanzó aproximadamente una semana más temprana en los animales que recibieron T2 (alfalfa + afrechillo).

Palabras Clave: Cuy, edad óptima de beneficio, alfalfa, afrechillo.

\section{Titroluccion}

El cuy doméstico (Cavia porcellus) representa una fuente valiosa de carne en las zonas rurales del país, siendo también muy apreciada en las zonas urbanas. La crianza del cuy se realiza mayormente en forma tradicional con escasas innovaciones técnicas; usualmente es una crianza familiar que permite al campesino aprovechar residuos alimenticios y forrajes disponibles en las zonas rurales para transformarlos en carne, que de otra manera se desaprovecharían. En línea con la necesidad de contribuir a mejorar la crianza y explotación del cuy se requiere evaluar alternativas alimenticias que sean económicas. En esta consideración el uso de algún suplemento sería beneficioso, de allí el interés de evaluar el afrechillo de trigo como suplemento alimenticio en el engorde de cuyes.

\section{Mhteriales y Métodos}

\section{Ubicación}

El estudio se realizó en laEstación Experimental del IVITA - El Mantaro durante los meses de diciembre de 1998 y abril de 1999. Este centro experimental está en el distrito de El Mantaro, provincia de Jauja, departamento de Junín; a una altitud de 3,320 $\mathrm{m}$, con una temperatura ambiental que oscila entre $19.9^{\circ} \mathrm{Cy}-1.8^{\circ} \mathrm{C}$ y una precipitación pluvial promedio anual de $770 \mathrm{~mm}$.

\section{Animales}

Se emplearon 40 gazapos (20 machos y 20 hembras), todos procedentes de madres primerizas, destetados a una edad promedio de 14,5 días, los que fueron divididos en dos grupos iguales y asignados al azar a los siguientes tratamientos:

\section{T1: Alfalfa fresca a discreción.}

T2: Alfalfa fresca más afrechillo a discreción.

\begin{abstract}
Alimentos
Se dispuso de $3000 \mathrm{~m}^{2}$ de alfalfa para corte, variedad Ranger, que se cosechó manualmente antes de la floración, suministrándose a los animales luego del oreado de 17 horas aproximadamente. Se compró $100 \mathrm{~kg}$ de afrechillo procedente de la planta molinera ubicada en el Distrito de Ataura, Provincia de Jauja. Se determinó la composición nutricional de ambos alimentos mediante análisis proximal (Cuadro 1).
\end{abstract}

\section{Manejo de la alimentación}

La alfalfa se ofreció 2 veces por día (9:00 y 14:00 horas), sobre una pequeña plancha de madera en una cantidad inicial de $600 \mathrm{~g}$ de alfalfa verde por día por unidad experimental, que se fue incrementando de acuerdo al consumo. El afrechillo fue administrado una vez por día (9:00 horas), en recipientes de arcilla, en una cantidad inicial 
Cuadro 1. Análisis proximal de alfalfa y afrechillo (base seca,\%).

\begin{tabular}{lcc}
\hline Composición & Alfalfa & Afrechillo \\
\hline Humedad & 78.66 & 6.52 \\
Materia seca & 21.34 & 93.48 \\
Proté́nas & 25.33 & 14.66 \\
Grasas & 4.67 & 2.88 \\
Fibra cruda & 34.06 & 14.98 \\
Ext. no nitrog. & 30.95 & 56.98 \\
Cenizas & 4.99 & 10.56 \\
\hline
\end{tabular}

de $80 \mathrm{~g}$ por día por unidad experimental, que también se fue incrementando de acuerdo al consumo; al día siguiente se pesaba el alimento no consumido y se volvía a ofrecer alimento fresco. La limpieza de la cama (paja) y desinfección de pozas se hizo cada cuatro semanas.

\section{Variables de respuesta}

Las variables de respuesta evaluadas fueron: Consumo de alimento semanal en gramos de materia seca, ganancia de peso semanal, conversión alimenticia, costos de producción (en Nuevos Soles S/.) y relación beneficio/costo.

\section{Determinación del momento óptimo eco- nómico de beneficio.}

De acuerdo al método descrito por Bautista (1974), se siguieron los siguientes pasos:

- Determinación de la ecuación de regresión: consumo de alimento $(\mathrm{X})$ - peso vivo (Y). Para lo cual se emplearon los datos del experimento: consumo de alimento semanal ( $\mathrm{g}$ de materia seca) y peso vivo promedio semanal (g), que se registraron hasta la semana 16. Los coeficientes de regresión se determinan por el método de DOOLITLE.

- Determinación de la ecuación de regresión: edad (X) - peso vivo (Y). Para este caso se requieren los datos del experimento: consumo de alimento semanal ( $g$ de materia seca) y peso vivo promedio semanal (g). Los coeficientes de regresión se determinan por el método de DOOLITLE.

- Estimación de la cantidad de alimento consumido en función de la ecuación de regresión: consumo de alimento peso vivo (ecuación de predicción). Se sabe además que el incremento de peso vivo por unidad de alimento equivale al producto marginal (Pma) que, a su vez, es la primera derivada de la función de producción (ecuación de predicción establecida), de donde el Pma puede expresarse en función del consumo de alimento. Para estimar la cantidad de gramos de alimento en materia seca a consumir por semana a un precio dado, podemos obtener una ecuación, donde el Pma multiplicado por el valor del $\mathrm{kg}$ de peso vivo del cuy es igual al precio del $\mathrm{kg}$ de alimento.

- Determinación del peso vivo (g) cuando el consumo de alimento máximo semanal 
sea el hallado en c, se usa la ecuación de regresión: consumo de alimento peso vivo, donde se reemplaza el valor de X para obtener Y.

- Utilización de la ecuación de regresión: edad - peso vivo, nos sirve para determinar en que tiempo de engorde se obtiene el peso hallado en $\mathrm{d}$.

Las variables de respuesta fueron comparadas entre tratamientos y entre sexos usando la prueba de $t$ de student pareado (Steel y Torrie, 1992).

\section{Resnlados y Diseusion}

Las edades óptimas de beneficio y los resultados de las variables de respuesta se presentan en el Cuadro 2. Puede notarse que la respuesta en $\mathrm{T} 2$ fue mejor que en $\mathrm{T} 1$, en todas la variables analizadas. En cuanto a la comparación de los sexos, los machos tuvieron mejor respuesta aunque sin mostrar diferencias estadísticas significativas
$(\mathrm{P}>0.05)$. El mayor consumo de alimento de T2 probablemente se explique por el hecho de disponer de dos tipos de alimento. Lógicamente un mayor consumo permite una mayor ganancia de peso, adicionalmente el T2 tuvo un mejor ICA respecto a T1, entonces al alimentar cuyes con alfalfa más afrechillo existe una buena complementación de nutrientes y se refleja en la conversión alimenticia.

Los resultados del presente estudio son mejores que los informados previamente. Así, se señala consumos de alfalfa en cuyes machos que varían de 40 a $50 \mathrm{~g} / \mathrm{cuy} /$ dia, y para hembras de 40.9 a $43 \mathrm{~g}$ (Valer et al., 1986; Huaras y Cook, 1989). Por otro lado, cuyes machos alimentados con alfalfa han logrado ganancias de peso de 4.4 a 8 g/cuy/día (Bernal, 1972; Arroyo, 1974; Valer et al., 1986; Quispe et al., 1994). Los índices de conversión alimenticia(ICA) para cuyes machos alimentados con alfalfa van de 4.6 a 15 (Bernal, 1972; Huaras y Cook, 1989). Con respecto a los conversión alimenticia, nuestros resultados pueden

Cuadro 2. Variables de respuesta y edad óptima de beneficio en cuyes alimentados con alfalfa fresca (T1) $v s$. alfalfa fresca + afrechillo (T2).

\begin{tabular}{|c|c|c|c|c|c|c|}
\hline \multirow[t]{2}{*}{ Variables evaluadas } & \multicolumn{2}{|c|}{$\mathrm{T} 1$} & \multirow{2}{*}{$\begin{array}{l}\text { Prome- } \\
\text { dio }\end{array}$} & \multicolumn{2}{|c|}{$\mathrm{T} 2$} & \multirow{2}{*}{$\begin{array}{c}\text { Prome- } \\
\text { dio }\end{array}$} \\
\hline & Macho & Hembra & & Macho & Hembra & \\
\hline Pesos iniciales & 352.5 & 333.4 & 343.0 & 334.0 & 321.6 & 328.0 \\
\hline $\begin{array}{l}\text { Consumo promedio g/MS/ } \\
\text { animal/d. }\end{array}$ & 57.0 & 53.6 & 55.3 & 59.6 & 59.3 & 59.5 \\
\hline $\begin{array}{l}\text { Ganancia de peso promedio, } \\
\text { g/animal/d. }\end{array}$ & 8.6 & 7.5 & 8.1 & 9.4 & 9.2 & 9.3 \\
\hline $\begin{array}{l}\text { Indice de conversión } \\
\text { alimenticia }\end{array}$ & 6.6 & 7.1 & 6.8 & 6.2 & 6.4 & 6.3 \\
\hline $\begin{array}{l}\text { Costo de producción hasta la } \\
\text { edad óptima de saca en S/. }\end{array}$ & 4.31 & 4.32 & 4.32 & 4.08 & 4.13 & 4.11 \\
\hline Relación beneficio/costo & 1.94 & 1.73 & 1.84 & 2.00 & 1.95 & 1.98 \\
\hline $\begin{array}{l}\text { Edad óptima económica de } \\
\text { beneficio en semanas }\end{array}$ & 12.87 & 12.94 & 12.91 & 11.75 & 11.99 & 11.87 \\
\hline
\end{tabular}


ubicarse en un rango intermedio con respecto a los resultados obtenidos por otros estudios. Estas diferencias son el resultado de la suma de factores influyentes, considerándose al potencial genético (Chauca y Zaldívar, 1985) como uno de los más importantes, además de factores alimenticios como la densidad energética del alimento, aporte nutricional, cantidad ofrecida, digestibilidad y estadío vegetativo en caso de ser forraje.

El costo de producción fue determinado considerando una capacidad de 1000 cuyes alimentados con alfalfa (Cuadro 3 ), inicialmente se determinan los costos y gastos y en función de estos se estima el costo de producción por cuy y por día. Y para calcular el costo de producción de un cuy en edad óptima de beneficio (Cuadro 4) además de conocer el costo de producción/ cuy/día, se debe conocer también el valor inicial del cuy. En cuanto a los tratamientos los costos de alimentación son iguales. Los resultados de costos de producción y relación beneficio costo por tratamiento se muestran en el Cuadro 2. El costo de producción/cuy/día es relativamente bajo, pues la capacidad que se considera en su determinación (1000 cuyes) reduce los costos; además, no se considera extrapolable para crianzas de menor capacidad y tecnificación, por que habría diferencias en los costos, principalmente de instalaciones, respecto a otros sistemas de crianza. Al no diferir los costos de alimentación entre tratamientos permite acceder al uso del afrechillo como suplemento. El menor costo de producción y por lo tanto la relación beneficio costo más favorable en T2 vs. T1, se explica en gran medida por la edad menor de beneficio de los animales.

Cuadro 3. Determinación de los costos de producción (S/.) considerando recursos para una capacidad de 1000 cuyes.

\begin{tabular}{llll}
\hline Costos & Monto & Vida útil (años) & Costo anual \\
\hline
\end{tabular}

A. Costo de Producción

$\begin{array}{lccc}\text { Alimentación }^{- \text {Terreno }}{ }^{1} & 50000 & \text { Indefinido } & 600 \\ \text { - Siembra de pasto }^{- \text {Mantenimiento de alfalfa }} & 4500 & 5 & 900 \\ \text { Instalaciones }^{\text {- Galpón y pozas }} & 1620 & 1 & 1620 \\ \text { Herramientas } & 40000 & 30 & 1333 \\ \text { Mano de obra } & 1458 & 5 & 291.6 \\ \text { Medicinas } & 5475 & - & 5475 \\ & 300 & 1 & 300\end{array}$

B. Gastos

$\begin{array}{lrrr}\text { Administrativos } & 150 & - & 150 \\ \text { Otros gastos } & 50 & - & 50\end{array}$

\begin{tabular}{ll}
\hline Costo total anual & 10719.60 \\
\hline Costo total/cuy/día & \\
\hline
\end{tabular}

En el costo anual del terreno se considera el impuesto predial anual.

${ }^{2}$ El costo de producción total no incluye el valor inicial del cuy. 
Cuadro 4. Costo total de cuyes en edad óptima de beneficio por tratamiento (S/.).

\begin{tabular}{lcccc}
\hline \multirow{2}{*}{ Tratamiento } & \multicolumn{3}{c}{ T1 } & \multicolumn{2}{c}{ T2 } \\
\cline { 2 - 5 } & Macho & Hembra & Macho & Hembra \\
\hline Valor inicial del cuy (gazapo) & 2.07 & 2.07 & 2.07 & 2.07 \\
Costo de producción hasta la saca/cuy & 2.24 & 2.25 & 2.01 & 2.06 \\
\hline Costo total/cuy en edad óptima de beneficio & 4.31 & 4.32 & 4.08 & 4.13 \\
\hline * El costo de producción hasta la saca se determina en función a las edades óptimas de beneficio de cada \\
$\begin{array}{l}\text { tratamiento. }\end{array}$
\end{tabular}

Los resultados presentados en el Cuadro 5, son los límites que hacen que el valor del producto marginal se iguale al precio marginal del alimento. El uso del afrechillo adelanta aproximadamente una semana la edad óptima de beneficio de un engorde logrado con una alimentación de alfalfa.

El sistema de comercialización por unidad animal, independiente del peso puede constituir un obstáculo al momento de realizar el análisis económico, sin embargo el precio de S/. 8.00 dado para animales de aproximadamente un $\mathrm{kg}$ de peso vivo, y los pesos esperados del presente estudio muy cercanos al kg, permiten atribuir utilidad práctica a los datos señalados en el Cuadro 5.

\section{Conelwiones}

- La edad óptima económica de beneficio de cuyes machos y hembras alimentados con alfalfa fue 12.9 semanas y de los alimentados con alfalfa más afrechillo como suplemento fue 11.8 para machos y 12.0 semanas para hembras. Estos resultados se cumplen cuando el precio del $\mathrm{kg}$ de materia seca de alimento cuesta $S / .0 .36$ y el kg de peso vivo de cuy se comercializa a S/. 8.00.

Cuadro 5. Edad óptima económica de beneficio, consumo y peso esperados y costos de alimento y por kg de cuy.

\begin{tabular}{|c|c|c|c|c|}
\hline \multirow{2}{*}{ Parámetros } & \multicolumn{2}{|c|}{$\mathrm{T} 1$} & \multicolumn{2}{|c|}{$\mathrm{T} 2$} \\
\hline & Macho & Hembra & Macho & Hembra \\
\hline $\begin{array}{l}\text { Edad óptima de beneficio, } \\
\text { sem. }\end{array}$ & 12.87 & 12.94 & 11.75 & 11.99 \\
\hline $\begin{array}{l}\text { Consumo de materia seca } \\
\text { esperado, g }\end{array}$ & 567.6 & 529.7 & 545.3 & 573.4 \\
\hline Peso vivo esperado, $\mathrm{g}$ & 1045.7 & 935.1 & 1025.1 & 1007.9 \\
\hline $\begin{array}{l}\text { Costo del } \mathrm{kg} \text { de } \mathrm{MS} \text { del } \\
\text { alimento, } \mathrm{S} / \text {. }\end{array}$ & 0.36 & 0.36 & 0.36 & 0.36 \\
\hline $\begin{array}{l}\text { Costo del } \mathrm{kg} \text { de peso vivo } \\
\text { del cuy, S/. }\end{array}$ & 8.00 & 8.00 & 8.00 & 8.00 \\
\hline
\end{tabular}


- En la alimentación de alfalfa más afrechillo, el consumo fue mayor, el afrechillo constituyó aproximadamente el $10 \%$ del consumo total de materia seca.

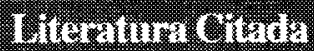

1. Arroyo, O. 1974. Uso de la alfalfa verde y el heno de alfalfa en el engorde de cuyes. En: Investigaciones en Cuyes. Vol. 1. Universidad Nacional del Centro del Perú. Huancayo. Perú. p. 23-28.

2. Bautista, A. 1974. Determinación de la edad óptima de comercialización y selección de cuyes (Cavia porcellus). Tesis para optar el Título de Ingeniero Zootecnista. Universidad Nacional Agraria La Molina, Lima. Perú. 59 p.

3. Bernal, S. 1972. Crecimiento y engorde de cuyes bajo tres sistemas de alimentación: alfalfa verde, alfalfa verde más concentrado y sorgo más concentrado. Tesis para optar el Título de Ingeniero Zootecnista. Universidad Nacional Pedro Ruiz Gallo, Lambayeque. Perú. 61 p.
4. Chauca, L. y M. Zaldívar. 1985. Investigación realizada en nutrición, selección y mejoramiento de cuyes en el Perú. INIPA. Sub Dirección de Crianzas. Lima. 2:30.

5. Huaras, U. y F. Cook. 1989. Cuatro raciones en el engorde de cuyes destetados. En: Resumen de la XII Reunión Científica Anual de la Asociación Peruana de Producción Animal (APPA). Lima. Perú. p. 114.

6. Quispe, C.; J. Castro y D. Chirinos. 1994. Engorde de cuyes a base de alfalfa y Phalaris tuberoarundinacea. En: Serie Informe Técnico $N^{\circ} 6$ - 94: Investigaciones en cuyes. Resúmenes de ALPA (1968-1990) y APPA (19761993). INIA-CIID. Lima. Perú. p. 132.

7. Steel, R.G.D. y J.H. Torrie. 1992. Bioestadística: Principios y Procedimientos. 2da. Ed. McGraw Hill S.A. México. $633 \mathrm{p}$.

8. Valer, A.; C. Blas y H. Taipe. 1986. Engorde de cuyes machos mejorados a base de dos raciones y evaluaciones de la carcasa. En: Resumen de la IX Reunión Científica Anual de la Asociación Peruana de Producción Animal (APPA). Tingo María. Perú. p. N-13. 